\title{
Evaluation of Pulmonary and Extrapulmonary Tuberculosis in Immunocompetent Adults: A Retrospective Case Series Analysis
}

\author{
Selma Ates Guler ${ }^{a} \quad$ Fulsen Bozkus $^{\mathrm{b}}$ Mehmet Fatih Incic Omer Faruk Kokoglu ${ }^{\mathrm{a}}$ \\ Hasan Ucmak ${ }^{a}$ Sevinc Ozden ${ }^{d}$ Murvet Yuksel ${ }^{c}$ \\ Departments of ${ }^{a}$ Infectious Diseases, ${ }^{b}$ Chest Diseases and ${ }^{\mathrm{c}}$ Radiology, Faculty of Medicine, Kahramanmaras Sutcu \\ Imam University, and d Department of Microbiology, Necip Fazil State Hospital, Kahramanmaras, Turkey
}

\section{Key Words}

Tuberculosis · Extrapulmonary tuberculosis .

Clinical features

\begin{abstract}
Objective: It was the aim of this study to evaluate the demographic factors and clinical features of extrapulmonary tuberculosis (EPTB) compared to those of pulmonary tuberculosis (PTB) among adult immunocompetent patients. Subjects and Methods: A total of 427 patients with clinically, radiologically and histopathologically confirmed TB were enrolled in the study, in our clinic at a tertiary care hospital in Turkey, during a 5-year period (2007-2012). Patient data were obtained retrospectively. Among the 427 patients, 55 patients with both PTB and EPTB and who were using steroids or had taken immunosuppressive drugs were excluded from the study. Results: Of the 372 patients, 227 (61\%) were males and 168 (45.2\%) had EPTB; 204 (54.8\%) patients had PTB. The most frequent sites of EPTB were the lymph nodes $(n=45,12.1 \%)$, pleura $(n=40,10.7 \%)$ and brain $(n=7,1.8 \%)$. The most common symptoms were cough $(n=174,46.7 \%)$, night sweats $(n=127,34.1 \%)$ and fever $(n=123,33 \%)$. Compared to EPTB patients, PTB patients were less likely to have received Bacillus Calmette-Guérin vaccination (odds ratio $0.41,95 \%$ confidence interval $0.2-0.63 ; p<0.001)$. Eightyone (48.2\%) of the EPTB and 146 (71.6\%) of the PTB patients
\end{abstract}

were males. Pulmonary involvement was more common among men $(n=146,71.6 \%)$ than among women $(n=58$, $28.2 \% ; p=0.000)$. Conclusion: There was a high incidence of EPTB in our study. Early diagnosis of EPTB is crucial for treatment, and atypical presentations of TB should be kept in mind for immunocompetent patients living in endemic areas. Females especially should be investigated for EPTB.

(c) 2014 S. Karger AG, Basel

\section{Introduction}

Tuberculosis (TB) is a major global health problem and may appear as a multisystem disease [1]. It is a chronic necrotizing bacterial infection characterized by the presence of granulomatous lesions, caused by the Mycobacterium tuberculosis complex. This includes $M$. africanum, M. microti, M. tuberculosis, and M. bovis. M. tuberculosis is responsible for $97-99 \%$ of disease development [2].

TB has two sequential processes: infection and active disease. Active disease can develop during any period of life in approximately $10 \%$ of the subjects who have been infected with the TB bacillus. The disease may involve any or all organs, but the lungs are the most commonly involved $(85 \%)$, and $50 \%$ of untreated patients die within 5 years after the initial infection [3]. According to the TB report of the World Health Organization in 2011, 39\% of

\begin{tabular}{ll}
\hline KARGER 125/s & $\begin{array}{l}\text { ( ) 2014 S. Karger AG, Basel } \\
1011-7571 / 14 / 0241-0075 \$ 39.50 / 0 \quad \text { Karger }\end{array}$ \\
$\begin{array}{l}\text { E-Mail karger@karger.com } \\
\text { www.karger.com/mpp }\end{array}$ & $\begin{array}{l}\text { This is an Open Access article licensed under the terms of the } \\
\text { Creative Commons Attribution-NonCommercial 3.0 Un- } \\
\text { ported license (CC BY-NC) (www.karger.com/OA-license), } \\
\text { applicable to the online version of the article only. Distribu- } \\
\text { tion permitted for non-commercial purposes only. }\end{array}$
\end{tabular}

Dr. Selma Ates Guler

Faculty of Medicine, Kahramanmaras Sutcu Imam University

Hastane cad. No. 50

TR-46000 Kahramanmaras (Turkey)

E-Mail selmaguler38@ hotmail.com 
TB patients in Turkey had extrapulmonary TB (EPTB) [4]. Of all the possible types of TB, the most common is pulmonary TB (PTB), which has great epidemiological significance due to its extremely contagious nature [5]. The proportion of patients with EPTB relative to those with PTB varies among countries and depends on associated diseases and ethnicity [6].

EPTB involvement tends to increase in frequency if the immune system is compromised [7].

Mycobacteria invade many organs during primary infection, but these foci remain dormant (without disease development) if the host has an effective immune system. Reactivation is accelerated in patients with latent diseases, under conditions where the immune system is suppressed. The risk of EPTB and mycobacteremia increases with advancing immunosuppression. Extrapulmonary involvement can be seen in $>50 \%$ of patients with concurrent AIDS and TB $[5,8-10]$. EPTB includes TB of organs other than the lung parenchyma, such as the lymph nodes, pleura, abdomen, genitourinary tract, gastrointestinal tract, skin, joints and bones, or meninges [8]. The diagnosis of EPTB is more difficult than that of PTB [8]. Its increasing incidence and severe sequelae due to the delay in diagnosis lead to a significant decrease in labor force [11].

The aim of this study was to evaluate demographic factors and clinical features of EPTB and compare them with those of PTB among adult immunocompetent patients $>15$ years of age.

\section{Subjects and Methods}

We studied the results of retrospective case series analyses of TB and EPTB. We included 427 patients who were clinically, radiologically and/or histopathologically diagnosed with active TB at Kahramanmaras State Hospital, Kahramanmaras Sutcu Imam University and Kahramanmaras Tuberculosis Dispensary between 2007 and 2012. Fifty-five patients with both PTB and EPTB or who were using steroids or had taken immunosuppressive drugs were excluded from the study. Patient data [gender, age, contact history, primary disease or recurrence, complaints at admission, Bacillus Calmette-Guérin (BCG) vaccination, symptoms, signs, involvement sites] were investigated retrospectively. In Turkey, since 2003, the Ministry of Health has performed pilot studies for the DOTS (Directly Observed Therapy Short-Course) strategy, and in 2006, the Tuberculosis Control Program was integrated into the primary health care system and the DOTS strategy expanded. The guidelines of the Ministry of Health are followed for the diagnosis of TB in all the clinical departments of our hospital.

Active TB was defined as an identification of M. tuberculosis through Ziehl-Neelsen acid-fast stain and culture in LowensteinJensen or BACTEC media in a tissue or infected specimen in culture-positive patients and it was defined with the help of clinical, laboratory, imaging and/or histopathological evidence of myco- bacterial infection in association with a positive tuberculin skin test reaction and/or a history of exposure to $\mathrm{TB}$ or a response to empirical antituberculosis treatment in culture-negative patients. Only lung parenchyma involvement was considered as PTB. EPTB was defined as the involvement of organs other than the lung parenchyma, such as the lymph nodes, pleura, abdomen, genitourinary tract, gastrointestinal tract, skin, joints and bones, meninges, and others. Our study was approved by the Ethical Committee of Kahramanmaras Sutcu Imam University.

Data were evaluated using SSPS 17.0. Differences in categorical variables between EPTB and PTB were compared and analyzed using the $\chi^{2}$ test. Odds ratios (ORs) and confidence intervals (CIs) were calculated to identify factors associated with EPTB compared to PTB. A 2 -sided $p$ value $<0.05$ was considered statistically significant.

\section{Results}

Of the 372 patients, 227 (61\%) were males and 145 $(38.9 \%)$ were females. The mean age of the patients with PTB was $39.2 \pm 17.1$ years (range 20-85), while that of the EPTB patients was $39.4 \pm 17.6$ years (range 18-78); the difference was not statistically significant $(\mathrm{p}=0.06)$. A history of contact with a case of TB was present in 39 patients $(10.4 \%)$, and 252 patients $(67.7 \%)$ were living at the city center. Six patients (1.6\%) had recurrent disease. Although data for the evaluation of the underlying disease were insufficient, the patients most commonly had chronic renal failure and diabetes mellitus. While 181 $(48.6 \%)$ of the patients had BCG vaccination, there was not vaccination in 191 patients (51.3\%). Miliary TB was observed in 5 patients (1.3\%).

Hilar lymph node involvement was encountered in 38 patients (10.2\%). Among all patients, 104 (27.9\%) were positive for acid-fast bacilli (AFB), and the diagnosis was confirmed in 173 cases (46.5\%) by histopathological examination. Among the 173 histopathologically diagnosed patients, only $22.4 \%$ were AFB positive. The culture (sputum, cerebrospinal/pleural/ascitic fluid) positivity ratio was $3.7 \%$ (14 patients). There were culture-positive results in $13 \mathrm{PTB}(3.4 \%)$ patients and 1 EPTB (0.2\%) patient. Common symptoms were: cough $(\mathrm{n}=174,46.7 \%)$, night sweats $(n=127,34.1 \%)$, fever $(n=123,33 \%)$, weight loss $(\mathrm{n}=111,29.8 \%)$, fatigue $(\mathrm{n}=95,25.5 \%)$, and hemoptysis $(\mathrm{n}=30,8.06 \%)$. Chest radiography was performed on all patients (EPTB and PTB) and was positive in $95.5 \%$ (195/204) of PTB patients. The diagnosis of EPTB was made according to the evaluation of histopathologic samples (124 patients) derived from pleural fluid analysis, or pleural biopsy (32 patients), fine-needle aspiration cytology (39 patients) and total excision (53 patients). 
Table 1. Comparison of pulmonary and extrapulmonary signs

\begin{tabular}{lccllr}
\hline & $\begin{array}{l}\text { Pulmonary } \\
(\mathrm{n}=204)\end{array}$ & $\begin{array}{l}\text { Extrapulmonary } \\
(\mathrm{n}=168)\end{array}$ & p value & OR & $95 \% \mathrm{CI}$ \\
\hline Gender F/M & $58(28.4) / 146(71.6)$ & $87(51.89) / 81(48.2)$ & 0.000 & 2.57 & $1.680-3.950$ \\
$\leq 25$ years & $62(62)$ & $38(38)$ & 0.001 & 0.243 & $0.105-0.559$ \\
$26-40$ years & $51(49)$ & $53(51)$ & 0.064 & 0.461 & $0.202-1.053$ \\
$>40$ years & $91(54.2)$ & $77(45.8)$ & 0.006 & 0.408 & $0.214-0.779$ \\
History & $24(11.8)$ & $10(6)$ & 0.07 & 0.478 & $0.222-1.030$ \\
City center & $140(68.6)$ & $112(66.7)$ & 0.738 & 1.094 & $0.707-1.692$ \\
Fever & $90(44.1)$ & $33(19.6)$ & 0.000 & 0.310 & $0.193-0.496$ \\
Weight loss & $72(35.2)$ & $39(23.1)$ & 0.011 & 0.554 & $0.350-0.877$ \\
Fatigue & $54(26.5)$ & $41(24.4)$ & 0.720 & 0.897 & $0.561-1.434$ \\
Night sweats & $93(45.6)$ & $34(20.2)$ & 0.000 & 0.303 & $0.190-0.483$ \\
Cough & $129(63.2)$ & $45(26.7)$ & 0.000 & 0.213 & $0.136-0.332$ \\
Hemoptysis & $24(11.7)$ & $51(30.3)$ & 0.003 & 0.524 & $0.241-0.566$ \\
AFB & $53(25.9)$ & $2(1.1)$ & 0.356 & 1.242 & $0.789-1.955$ \\
Recurrence & $4(1.9)$ & $124(73.8)$ & 0.007 & 0.164 & $0.37-0.730$ \\
Pathological diagnosis & $49(24.1)$ & $64(38)$ & 0.000 & 0.266 & $0.172-0.411$ \\
BCG & $117(57.3)$ & 0.000 & 0.418 & $0.202-0.634$ \\
\hline
\end{tabular}

Figures in parentheses are percentages. $\mathrm{F}=$ Female; $\mathrm{M}=$ male.

Thorax CT examination revealed PTB in 102 patients (50\%), and CT results were cavitation in 9 patients $(8.8 \%)$, consolidation in 41 patients (40.1\%), pleurisy in 39 patients $(38.2 \%)$, and fibrotic changes and sequelae in 11 patients (10.7\%).

Of the 372 patients, 204 (54.8\%) were diagnosed with PTB and $168(45.1 \%)$ with EPTB. Of the 204 PTB patients, $146(71.5 \%)$ were males and $58(28.4 \%)$ were females, whereas of the EPTB patients, 81 (48.2\%) were males and 87 (51.7\%) were females. While the PTB ratio was significantly higher in men, the EPTB ratio was significantly higher in women (OR 2.57, 95\% CI 1.68-3.95; $\mathrm{p}=0.000$ ). Pulmonary involvement was more prominent in cases with BCG vaccinations; 64 EPTB patients (38\%) and 117 PTB patients (57.3\%) had BCG vaccination, respectively (OR 0.41, 95\% CI 0.27-0.63; $\mathrm{p}=0.000$ ). Symptoms such as hemoptysis, weight loss, fever, night sweats, and cough were statistically more significant in patients with PTB compared to those with EPTB ( $\mathrm{p}=0.003, \mathrm{p}=$ $0.011, p=0.000, p=0.000$, and $p=0.000$, respectively). A comparison of pulmonary and extrapulmonary involvement is shown in table 1.

Extrapulmonary involvement was most common in the lymph nodes $(\mathrm{n}=45,12.9 \%)$ and pleura $(\mathrm{n}=40$, $10.7 \%$ ), followed by (in decreasing order) the brain, psoas muscle, bone, breast, skin, nasopharynx, fallopian tubes, ovary, pancreas, and the spleen. A distribution of the in- volvement sites of the EPTB cases is shown in table 2. EPTB was also commonly seen in the cervical, inguinal, mediastinal, axillary, paratracheal, and supraclavicular areas as well as observed as a fistula of the esophagus. Lymphadenopathy according to the involvement sites is shown in table 2.

\section{Discussion}

There was a high incidence of EPTB in our study (45.1\%). The most common sites involved were the lymph nodes and pleura, followed by (in decreasing order) the brain, psoas muscle, bone, breast, skin, nasopharynx, fallopian tubes, ovary, pancreas, and the spleen. The most common symptoms were cough, night sweats, and fever. While the ratio of PTB was significantly higher in men, the ratio of EPTB was significantly higher in women. Pulmonary involvement was more prominent in cases with $B C G$ vaccinations. For the diagnosis of $\mathrm{PTB}$, chest radiography, Mantoux, and BCG tests were done while EPTB was diagnosed using various diagnostic procedures.

The incidence of EPTB has been reported as 4.5-53.8\% in studies from Turkey [12-18]; in our study, there were $61 \%$ men, and the male/female ratio was 1.5 . This ratio is reported as 1.5/1.6 in the worldwide literature [15, 16, 19-23]. 
Table 2. Distribution of involvement sites in EPTB cases

\begin{tabular}{|c|c|c|}
\hline Involved area & $\mathrm{n}$ & $\%$ \\
\hline Lymph nodes & 45 & 12.0 \\
\hline Cervical & 38 & 9.2 \\
\hline Inguinal & 2 & 0.5 \\
\hline Mediastinum & 1 & 0.2 \\
\hline Axillary & 1 & 0.2 \\
\hline Paratracheal & 1 & 0.2 \\
\hline Mediastinal LAP & 1 & 0.2 \\
\hline Supraclavicular & 1 & 0.2 \\
\hline Pleural & 40 & 10.8 \\
\hline \multicolumn{3}{|l|}{ Abdominal } \\
\hline Peritoneum & 4 & 1.1 \\
\hline Omentum & 2 & 0.5 \\
\hline Intestines & 4 & 1.1 \\
\hline \multicolumn{3}{|l|}{ Urogenital } \\
\hline Kidney & 3 & 0.8 \\
\hline Ovarian tubes & 2 & 0.5 \\
\hline Testicles & 1 & 0.3 \\
\hline Ovary & 2 & 0.5 \\
\hline \multicolumn{3}{|l|}{ Osteoarticular } \\
\hline Vertebra & 7 & 1.9 \\
\hline Spondylodiscitis & 2 & 0.5 \\
\hline Wrist & 1 & 0.3 \\
\hline \multicolumn{3}{|l|}{ Skin and soft tissue } \\
\hline Breast & 11 & 0.3 \\
\hline Skin & 3 & 0.8 \\
\hline Psoas & 3 & 0.8 \\
\hline Nasopharynx & 1 & 0.3 \\
\hline CNS & 7 & 1.9 \\
\hline
\end{tabular}

LAP = Lymphadenopathy; CNS = central nervous system.

While TB is encountered in advanced ages in developing countries, the majority of our cases (in both groups) were in the 20- to 40-year age group. These data are consistent with results of other studies from Turkey $[12,16]$. Although the role of gender in TB is not yet clearly defined, extrapulmonary involvement was reported to be more common among women $[6,14]$. Our results were similar to those reports. In the EPTB cases, $25-65.8 \%$ of patients showed a history of contact with a case of TB [12, 18]. The low contact ratio found in our study may be due to the low awareness of our patients. In earlier studies conducted in other regions of Turkey, the most frequently seen forms of EPTB were pleural TB, lymphatic TB, and central nervous system TB [14].

Lymphadenitis was the most frequent type of EPTB in our study. Khan et al. [24] reported that of 1,548 TB cases, 109 patients $(7.0 \%)$ had TB lymphadenitis. Cervical ade- nopathy was the most common cause, but inguinal, axillary, mesenteric, mediastinal, and intramammary involvements have also been reported [18]. In our study, while cervical lymphadenopathy was most commonly encountered, atypical involvement areas (inguinal, axillary, and mesenteric lymphadenopathies) were also observed. Those were diagnosed by histopathological examination of the dissected lymph nodes. The results of our study are comparable with two other studies $[25,26]$, which have reported that lymph nodes are involved in almost half of the EPTB cases. Our distribution of EPTB differs from previous studies. For example, Yang et al. [6] have reported that the most common involvement sites are bone/ joints and lymph nodes in the United States, whereas the genitourinary system and skin are the most common involvement sites in a report from Hong Kong [26]. This may be attributed to ethnic differences or underlying associated diseases [8].

M. tuberculosis culture is the definitive method of diagnosing an active infection and should be done whenever possible. In clinical practice, a positive culture is only obtained in about two thirds of the pulmonary TB cases and in fewer than half of the EPTB patients [9]. In our study, the positive culture ratio was low $(3.7 \%)$ in both groups. Reasons may be the lack of technical skills, which prohibits performing a culture analysis in every healthcare unit and the low rates of bacterial growth in these cultures. The sputum smear was positive in more than half of the PTB cases, although not always with the initial specimen. A positive sputum smear, usually in extensive disease, provides additional evidence for confirming the diagnosis. At least three specimens are recommended for greater sensitivity [2] and at least three sputum samples should be obtained routinely if TB is suspected [27]. In our study, AFB positivity was detected in $44.3 \%$ of PTB and in $34.8 \%$ of EPTB patients. The high AFB positivity ratio among extrapulmonary cases may be due to the AFB staining of the tissue cultures, as well as due to the frequent use of histochemical methods in diagnosing extrapulmonary involvement. Hemoptysis, weight loss, night sweats, and fever were more frequent among cases with pulmonary involvement, as expected, than among cases with extrapulmonary involvement. The BCG vaccine contains an $M$. bovis species which has all of the structural characteristics of a TB bacillus, but without the disease-forming ability [28]. Among patients who have received BCG vaccination, severe forms of TB (like miliary TB and TB meningitis) were very rarely encountered [29]. In our study, there have been doubts about whether the BCG vaccine can
Ates Guler/Bozkus/Inci/Kokoglu/ Ucmak/Ozden/Yuksel 
prevent latent infection in subjects with previous vaccinations. The BCG vaccination rate was higher in patients with pulmonary involvement $(57.3 \%)$ than in those with extrapulmonary involvements (38\%), and it could thus be concluded that the BCG vaccine has a low potency in the prevention against latent infection or that the infection was limited to lungs of patients subject to previous BCG vaccination. However, this conclusion should be investigated in a more comprehensive epidemiological study. Our study has some methodological limitations. The information was obtained retrospectively from patient files and was probably not as complete and accurate as the data collection which was done prospectively.

\section{Conclusion}

EPTB frequency was high in our study. EPTB still remains a significant cause of morbidity and mortality. Thus, early diagnosis and initiation of appropriate treatment are important for reducing morbidity and mortality in patients with EPTB. Atypical presentations of TB should not be ignored in immunocompetent patients living in endemic areas. Women especially should be investigated for EPTB, and BCG vaccination should be encouraged.

\section{Disclosure Statement}

There are no conflicts of interest.

\section{References}

$\checkmark 1$ Murthy KAS, Kiran HS, Cheluvaraj V, et al: Young lady with a swelling over the back: a rare case of tuberculosis. Iran J Med Sci 2011; 36:318-321.

2 Fitzgerald DW, Sterling TR, Haas DW: Mycobacterium tuberculosis; in Mandell GL, Bennett JE, Dolin R (eds): Mandell, Douglas, and Bennett's Principles and Practice of Infectious Diseases, ed 7. New York, Churchill Livingstone, 2009, pp 3129-3133.

3 Haas DW, Des Prez RM: Mycobacterium tuberculosis; in Mandell GL, Bennett JE, Dolin R (eds): Mandell, Douglas, and Bennett's Principles and Practice of Infectious Diseases, ed 5. New York, Churchill Livingstone, 1995, pp 2213-2243.

4 World Health Organization: World Health Organization global tuberculosis report. http://www.who.int/tb/publications/global_ report/archive/en/.

5 Al Otaibi F, El Hazmi MM: Extra-pulmonary tuberculosis in Saudi Arabia. Indian J Pathol Microbiol 2010;2:227-231.

-6 Yang Z, Kong Y, Wilson F, et al: Identification of risk factors for extrapulmonary tuberculosis. Clin Infect Dis 2004;38:199-205.

7 Shafer RW, Edlin BR:Tuberculosis in patients infected with human immunodeficiency virus: perspective on the past decade. Clin Infect Dis 1996;22:683-704.

8 Lin JN, Lai CH, Chen YH, et al: Risk factors for extra-pulmonary tuberculosis compared to pulmonary tuberculosis. Int J Tuberc Lung Dis 2009; 13:620-625.

-9 Golden MP, Vikram HR: Extrapulmonary tuberculosis: an overview. Am Fam Physician 2005;9:1761-1768.

10 Aaron L, Saadoun D, Calatroni I, et al: Tuberculosis in HIV-infected patients: a comprehensive review. Clin Microbiol Infect 2004;
10:388-398.

11 Dwyer DE, Collignon PJ, Macleod C, et al: Extrapulmonary tuberculosis. A continuing problem in Australia. Aust NZ J Med 1987;17: 507-511.

12 Sevgi DY, Derin O, Alpay AS, et al: Extrapulmonary tuberculosis: 7 year-experience of a tertiary center in Istanbul. Eur J Intern Med 2013;24:864-867.

13 Akgun M, Kaynar H, Saglam L, et al: Clinical and social characteristics of the patients with tuberculosis in Eastern Anatolia. Tuberk Toraks 2006;54:349-354.

14 Gunal S, Yang Z, Agarwal M, et al: Demographic and microbial characteristics of extrapulmonary tuberculosis cases diagnosed in Malatya, Turkey, 2001-2007. BMC Public Health 2011;11:154.

15 Ozvaran MK, Baran R, Tor M, et al: Extrapulmonary tuberculosis in non-human immunodeficiency virus-infected adults in an endemic region. Ann Thorac Med 2007;2:118121.

16 Oztop A, Unsal I, Gunay T, et al: The study of evaluation of tuberculous patients who registered in Kahramanlar tuberculous struggle dispensary. Tuberk Toraks 2000;48:243-247.

17 Cakir E, Erdem E, Ozlu N, et al: Demographic and microbial characteristics and drug resistance of childhood tuberculosis in Istanbul: analysis of 1,541 cases. J Infect Dev Ctries 2014;8:304-309.

18 Ilgazli A, Boyaci H, Basyigit I, et al: Extrapulmonary tuberculosis: clinical and epidemiologic spectrum of 636 cases. Arch Med Res 2004;35:435-441.

19 Varkey P, Jerath AU, Bagniewski SM, et al: The epidemiology of tuberculosis among primary refugee arrivals in Minnesota between
1997 and 2001. J Travel Med 2007;14:18

20 Mahendradhata Y, Syahrizal BM, Utarini A: Delayed treatment of tuberculosis patients in rural areas of Yogyakarta province, Indonesia. BMC Public Health 2008;8:393-399.

21 Nissapatorn V, Kuppusamy I, Sim BLH, et al: Pulmonary tuberculosis in a hospital setting: gender differences. Public Health 2006;120: 441-443.

22 Gonzalez OY, Adams G, Teeter LD, et al: Extra-pulmonary manifestations in a large metropolitan area with a low incidence of tuberculosis. Int J Tuberc Lung Dis 2003;7:11781185.

23 Holmes CB, Hausler H, Nunn P: A review of sex differences in the epidemiology of tuberculosis. Int J Tuberc Lung Dis 1998;2:96-104.

24 Khan HA, Sulaiman SAA, Muttalif AR, et al: Tuberculous lymphadenitis at Penang General Hospital, Malaysia. Med Princ Pract 2011;20:80-84.

25 Sreeramareddy CD, Panduru KV, Verma SC, et al: Comparison of pulmonary and extrapulmonary tuberculosis in Nepal - a hospitalbased retrospective study. BMC Infect Dis 2008;8:8

26 Musellim B, Erturan S, Sonmez Duman E, et al: Comparison of extra-pulmonary and pulmonary tuberculosis cases: factors influencing the site of reactivation. Int J Tuberc Lung Dis 2005;9:1220-1223.

27 Haney S, Stenton SC: Tuberculosis and the older patient. Rev Clin Gerontol 2010;20:8191.

28 Fine PE: Bacille Calmette-Guerin vaccines: a rough guide. Clin Infect Dis 1995;20:11-14.

29 Bass JB Jr, Farer LS, Hopewell PC, et al: Treatment of tuberculosis and tuberculosis infections in adults and children. American Thoracic Society and the Centers for Disease Control and Prevention. Am J Respir Crit Care Med 1994;149:1359-1374. 